
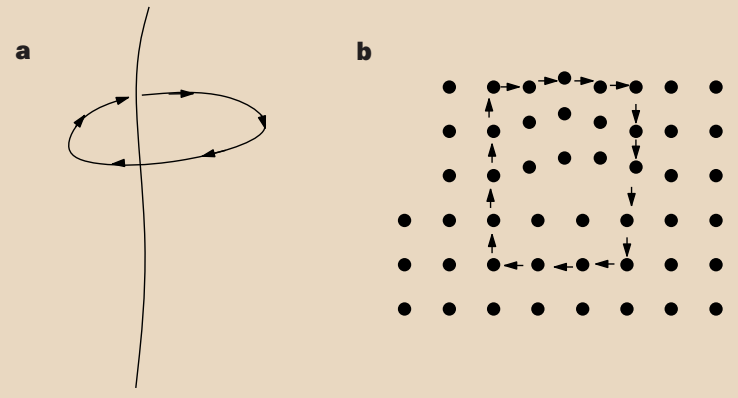

superfluid ${ }^{4} \mathrm{He}$, or for magnetic flux lines in superconductors.

Dislocations, however, are more complicated than their fluid counterparts. The measure of dislocation vorticity, $\vec{b}$, is a vector. So dislocations come in several different flavours depending upon the relative direction between $\vec{b}$ and the dislocation line. Dislocation cores can play tricks that are unfamiliar in fluids. They sometimes decide to split into two nearby partial dislocations, each of which carries only a fraction of the quantized vorticity $\vec{b}$ the crystal demands, with a thin sheet of slightly defective solid connecting the two lines.

Even greater complications arise when two dislocations of differing character head towards one another and collide. Each dislocation has a particular direction in which it prefers to travel. When two combine, they have as much chance of travelling further as two dogs with their hind legs tied together on a long leash trying to run through a thick forest. Lomer ${ }^{8}$ and Cottrell ${ }^{9}$ pointed out that dislocation pairs in common metals should indeed attract each other, lock up and become immobilized; such Lomer-Cottrell locks are frequently observed experimental$l y^{10}$. When they are mobile, dislocations explain the ability of crystals to flow. But as they lock up, they explain the fact that the more a crystal flows, the stiffer it becomes in resisting further deformation.

The main virtue of viewing a deforming crystal as an assembly of dislocations lies in the hope of ignoring almost all detailed information on locations of the atoms and describing the evolution of the crystal purely in terms of dislocation motion. In a mesoscopic description (that is, at scales of about $10^{-6} \mathrm{~m}$ ), dislocations are treated as curving lines that interact with one another, and with outside forces. Computer programs adopting this point of view have been written by several groups (including those of Kubin and Devincre, two of the authors of the new work $^{1}$ ), but have been hampered by some uncertainties. How fast should known forces make dislocations move? When they bind and interact, what force is needed to unlock them? How will the process happen?

As Bulatov et al. ${ }^{1}$ show, molecular dynamics simulations, following the motion of up to 100 million atoms under the influence of Newton's laws, are finally beginning
Figure 2 Vortices in fluids and solids. a, The circulation $\mathrm{k}$ around a vortex in a fluid is given by summing all fluid velocities around a loop. b, The analogous quantity in a crystal is given by summing arrows between nearest neighbours over a loop surrounding a dislocation; there are four on top and three on the bottom, so they do not all sum to zero. from the original pair, unzips the lock and forms a new dislocation stretching in a new direction - divorce and remarriage at the atomic scale.

Some features of the calculation are still schematic; for example, the force laws between atoms have been chosen more for computational efficiency than for maximum possible realism. However, given the interest in the geometry of dislocation motion, such details are not likely to be important, and what matters is the careful extraction of information from small scales, used as the foundation for calculations on larger scales. The ultimate goal of computer programs to mimic solids with full realism is not yet locked up, but progress is being made.

Michael Marder is in the Center for Nonlinear Dynamics and the Department of Physics, The University of Texas at Austin, Austin, Texas 78712, USA.

e-mail:marder@chaos.ph.utexas.edu

1. Bulatov, V., Abraham, F. F., Kubin, L., Devincre, B. \& Yip, S. Nature 391, 669-672 (1998).

2. Thomson, J. J. The Corpuscular Theory of Matter (Scribner's, New York, 1907).

3. Friedel, J. Dislocations (Addison Wesley, Reading, MA, 1964)

4. Hirth, J. P. \& Lothe, J. Theory of Dislocations 2nd edn (Wiley, New York, 1982).

Taylor, G. I. Proc. R. Soc. Lond. A 145, 362-404 (1934).

6. Orowan, E. Z. Phys. 89, 605-659 (1934).

Polanyi, M. Z. Phys. 89, 660-666 (1934).

8. Lomer, W. M. Phil. Mag. 42, 1327-1331 (1951).

9. Cottrell, A. H. Phil. Mag. 43, 645-647 (1952).

10. Ferret, P., Robinson, B. J., Thompson, D. A. \& Baribeau, J. M. Appl. Phys. Lett. 57, 2220-2221 (1990).

\title{
HiV vacoines \\ Viral envelope fails to deliver?
}

\section{Dani P. Bolognesi and Thomas J. Matthews}

$\mathrm{O}$ n 17 June 1994, the National Institutes of Health announced ${ }^{1}$ that it would continue - but not expand - clinical trials with two vaccines against the human immunodeficiency virus (HIV), which use recombinant viral gp120 subunits to raise an immunity to the virus. To some this decision was viewed as a major setback, given the resources that had been expended in the development and testing of these vaccines. But to others, who felt that to test products with questionable promise was risky, unjustifiably costly, and would have a negative effect on future vaccine trials, this outcome was more satisfying. These events highlight a continuing struggle to establish standards for a vaccine that are acceptable to scientists, vaccine developers, government officials and the communities affected by such trials.

Almost four years later, we have new information that raises more questions about the use of these two candidate vaccines $^{2-6}$. In the Journal of Virology, Connor et al. ${ }^{3}$ describe their analysis of 18 volunteers who became infected with HIV-1 while taking part in a phase II study of the two recombinant gp120 subunit vaccines. The authors searched for any indications that the vaccinated people dealt differently with their infections than did unvaccinated controls - but found none. In agreement with another study of a single case $e^{4}$, they concluded that even when the vaccine performed optimally (that is, when the full schedule of immunizations was given and

\begin{tabular}{|c|c|c|c|c|}
\hline Company & Subunit & Clade & Clinical trial & Status \\
\hline VaxGen & gp120 & B & Phase I/II - US & Started \\
\hline VaxGen & gp 120 & $\mathrm{E}$ & Phase I/I - Thailand & Imminent \\
\hline Chiron & gp120 & $\mathrm{E}$ & Phase I//l - Thailand & Imminent \\
\hline
\end{tabular}

A number of primary-isolate constructs are also under study, including oligomeric gp140 and gp160 constructs, pseudovirions, virus-like particles and DNA. 
the recipient became infected with HIV-1 when the immune responses were near peak levels), the host responses generated had no perceptible effects on the nature or progression of the infection.

Why did these vaccines fail? One intriguing possibility, as noted by Berman et al..$^{5}$, is that the subjects became infected with HIV-1 isolates bearing envelope proteins that differed from the vaccine immunogen at several neutralizing antibody-sensitive sites. But Connor et al. ${ }^{3}$ (and others ${ }^{6}$ ) reduced this possibility by comparing contemporary infections in matched controls. Therefore, vaccination with viral-envelope subunits alone did not exert overt selective pressure on the HIV-1 strains with which the patients became infected. Although this conclusion weakens the case for further development of these gp120 products, it is not possible to infer anything about their efficacy from these studies ${ }^{3,6}$ - except that the subunit vaccines are not $100 \%$ protective - because the results stem from relatively small studies.

The viral gp120 is one of two glycoproteins that make up the HIV outer envelope. It targets the virus to certain cells in the body by binding to specific cell receptors, and it initiates the early steps of virus entry into these targeted cells. Recombinant gp120 subunits emerged as candidates for vaccine development based on their ability to raise antibodies that effectively neutralize laboratory strains of HIV in vitro. Moreover, defined thresholds of such antibodies prevented infection of chimpanzees by the HIV strains from which the vaccines were derived. Studies for safety and immunogenicity in humans were successful, laying the foundations to test whether levels of neutralizing antibody correlated with immunity against HIV infection. But first, researchers tried to show that the neutralizing-antibody response would be effective against the viruses circulating in populations where the trial was to be carried out. In laboratory assays using field strains, however, no neutralizing activity was detectable ${ }^{7}$.

Why are laboratory strains of HIV sensitive to antibody neutralization, whereas primary viruses are resistant? There seem to be substantive differences between the envelope structures of these two viral phenotypes, which also use different co-receptors for infectivity. Phase I trials of several candidate vaccines that use the gp120 subunit derived from primary virus isolates are already underway (Table 1). But many researchers doubt that the simple substitution of one gp120 subunit for another will be enough to cause these constructs to act any differently from the early prototypes based on laboratory strains. Nor is there enough knowledge to design immunogens that can effectively induce neutralizing antibodies with sufficient potency and breadth against primary viruses. Insights to this end could emerge from increased understanding of the HIV envelope, its multimeric nature, the shielding of antibody targets by carbohydrate, and the structural transitions of HIV during receptor/co-receptor binding, fusion and entry into the target cell.

The main vaccine concept currently under development is a combination of a multicomponent canarypox viral vector and one of the original gp120 envelope subunits. Such combined vaccines induce detectable cytotoxic T lymphocytes in about $60 \%$ of vaccinated people ${ }^{8}$. These lymphocytes can recognize and lyse target cells infected by field strains, even from distantly related virus families (clades) ${ }^{9}$ — in contrast to the narrow specificity of the antibodies induced by the gp120 vaccines. The antibody responses induced by the canarypox vector alone are limited, but they are substantially improved when combined with the gp120 subunit boost. Although these antibodies do not neutralize primary HIV isolates, they induce antibody-dependent cell cytotoxicity, which is detectable in $50-70 \%$ of vaccinees. Moreover, T-cell proliferative responses are more potent and durable when the two vaccines are combined $^{8}$, and the response of CD4-positive $\mathrm{T}$ cells has been highlighted as a possible correlate for disease progression ${ }^{10}$.

The HIV-vaccine field remains at a crossroads. Development of an effective vaccine entails a proper balance between the growing information about HIV and empirical principles that have guided the successful production of vaccines against other agents. Correlates of protection, although useful in guiding preclinical studies, can only be established retrospectively from the results of appropriately designed clinical trials. Combining studies such as that of Connor et al. ${ }^{3}$ with sufficiently powerful clinical trials should allow the immunological and virological parameters that correlate with the success or failure of a vaccine to be dissected. Experiments are critical for the advancement - and, ultimately, development - of an effective vaccine against HIV.

Dani P. Bolognesi and Thomas J. Matthews are in the Department of Surgery, Duke University

Medical Center, PO Box 2926, Durham, North

Carolina 27710, USA.

\section{Cohen, J. Science 264, 1839 (1994).}

2. McElrath, J. et al. Proc. Natl Acad. Sci. USA 93, 3972-3977 (1996).

3. Connor, R. I. et al. J. Virol. 72, 1552-1576 (1998)

4. Kahn, J. et al. J. Infect. Dis. 171, 1343-1347 (1995).

5. Berman, P. et al. J. Infect. Dis. 176, 384-397 (1997).

6. Graham, B. et al. J. Infect. Dis. 177, 310-319 (1998).

7. Matthews, T. AIDS Res. Hum. Retroviruses 10, 631-632 (1994).

8. Crey, L. et al. 4th Conf. Retroviruses Opportunistic Infect. (in the press).

9errari, G. et al. Proc. Natl Acad. Sci. USA 94, 1396-1401 (1997)

10. Rosenberg, E. et al. Science 21, 1447-1450 (1997).

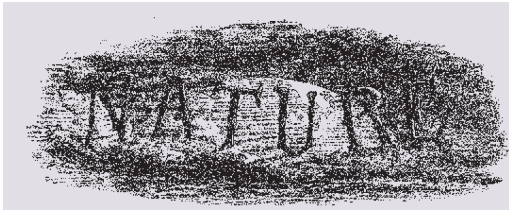

100 YEARS AGO

The War of the Worlds.

By H. G. Wells. Pp. 303.

(London: William Heinemann, 1898.)

Many writers of fiction have gathered material from the fairy-land of science, and have used it in the construction of literary fabrics, but none have done it more successfully than Mr. H. G. Wells. It is often easy to understand the cause of failure. The material may be used in such a way that there appears no connection between it and the background upon which it is seen; it may be so prominent that the threads with which it ought to harmonise are thrown into obscurity; or (and this is the worst of all) it may be employed by a writer whose knowledge of natural phenomena is not sufficient to justify his working with scientific colour. Mr. Wells makes none of these mistakes. Upon a groundwork of scientific fact, his vivid imagination and exceptional powers of description enable him to erect a structure which intellectual readers can find pleasure in contemplating. From Nature 10 February 1898.

\section{YEARS AGO}

A new high-altitude research laboratory for cosmic ray work at a height of 11,500 $\mathrm{ft}$. on the upper slopes of Monte Rosa was opened by the Italian Centre for Research in Nuclear Studies on January 11. The laboratory portion of the station at present consists of one large experimental room, and a small fully screened room for a Wilson cloud chamber. The equipment is very complete, and includes a three-phase $\mathbf{3 0}$ kW. power supply, a separate lighting supply, and two high-capacity battery sets with a petrol generator in case of main power failure. The station is also equipped for two-way direct radio contact with the parent laboratory at Rome, a useful facility at all times, but particularly necessary when the Laboratory is likely to be cut off from the outside world for days at a time in midwinter. ... The work at present going on in the Laboratory includes the exposure of nuclear plates, which in suitable weather can also be carried out up to $3,000 \mathrm{ft}$. above the Laboratory. Experiments on meson decay are also being carried on, and it is expected that later some Italian geneticists will be undertaking work at the station on mutations induced by cosmic rays. From Nature 14 February 1948. 